\title{
Significado del Riesgo de VIH/SIDA para Hombres Homosexuales en Medellín, Colombia
}

\section{Meaning of HIV/AIDS Risk for Gay Men in Medellín, Colombia}

\author{
Isabel Cristina Posada Zapata y Érika Tatiana Agudelo Olarte \\ Universidad de Antioquia
}

\begin{abstract}
Esta investigación buscó establecer el significado que tienen los hombres homosexuales sobre sus comportamientos de riesgo de contraer sexualmente el VIH/SIDA. Con un enfoque cualitativo, se entrevistó a 19 hombres homosexuales de la ciudad de Medellín, Colombia. Se utilizó la teoría fundamentada, partiendo del interaccionismo simbólico, para buscar la relación entre los significados y los comportamientos. Se desarrollaron procesos de codificación, categorización e interpretación. Los resultados muestran que (a) la clandestinidad de los encuentros sexuales hace que el riesgo se asuma como una forma de vivir el afecto y (b) existe una asociación inversa entre el afecto y el riesgo asumido: a mayor presencia de afecto mayor evitación del riesgo, consecuente con la información sobre prevención, y a mayor ausencia de afecto mayor disposición al riesgo, buscando el reconocimiento y negando la información sobre protección.
\end{abstract}

Palabras clave: homosexualidad, VIH/SIDA, exposición al riesgo, autonomía, afecto

This research aimed to establish the meaning that risk behaviors have for homosexual men in relation to contracting HIV/AIDS through sexual contact. Adopting a qualitative approach, 19 interviews were held with homosexual men residing in the city of Medellín, Colombia. Grounded theory, from the perspective of symbolic interactionism, was used to find the relationship between meanings and behaviors. Coding, categorization, and interpretation processes were conducted. Results show that (a) the clandestine nature of sexual encounters causes risk to be regarded as a way of experiencing affection and that (b) there is an inverse relationship between affection and risk: the stronger the affection the greater the risk avoidance, informed by prevention recommendations; the weaker the affection the greater the proneness to take risks, searching for recognition and denying the information about protective measures.

Keywords: homosexuality, HIV/AIDS, risk exposure, autonomy, affection

El fenómeno del virus de inmunodeficiencia humana (VIH)/SIDA se presenta como un grave problema de salud pública mundial, en el que no solamente se abarca el contexto natural de la enfermedad, sino un proceso que tiene un devenir en diversas dimensiones, como la social, económica y cultural. Las implicaciones que se han presentado en el mundo desde la aparición del virus rebasan el orden del campo biológico, haciendo pertinente el abordaje desde diferentes áreas de estudio que orienten la búsqueda para generar alternativas en los programas que intentan disminuir el número de infectados y las conductas de riesgo. Entre los efectos sociales por el VIH, algunos causan un mayor impacto, como los niños en situación de orfandad por la pérdida de sus padres, los servicios de salud desbordados, el hambre y el aumento de la escasez económica, debido a la falta de personas sanas para la producción de activos, el reforzamiento de las cadenas de pobreza y el aumento del estigma (Organización Mundial de la Salud, 2004).

En Colombia una de las poblaciones más afectadas por el SIDA son los varones homosexuales (Colombia, Ministerio de Salud y Protección Social \& Fondo de Población de las Naciones Unidas [UNFPA], 2012) que, por diversos factores (aspectos políticos para enmascarar esta población, priorización de fondos, tratado de su identidad, uso en estudios y publicaciones científicas), en Latinoamérica se han nombrado como hombres que tienen sexo con hombres (HSH; Estrada-Montoya, 2014) y que incluye tanto a hombres que se consideran

Isabel Cristina Posada Zapata y Érika Tatiana Agudelo Olarte, Facultad Nacional de Salud Pública, Universidad de Antioquia, Medellín, Colombia.

Érika Tatiana Agudelo Olarte pertenece ahora al Centro de Evaluación y Medición de la Facultad de Psicología (CESMIDE), Universidad CES, Medellín, Colombia.

El desarrollo del artículo fue apoyado por recursos de sostenibilidad CODI 2013-2014.

La correspondencia relativa a este artículo debe ser dirigida a Isabel Cristina Posada Zapata, Facultad Nacional de Salud Pública, Universidad de Antioquia, Calle 62, \# 52-59, Oficina 200, Medellín, Colombia. E-mail: isabel.posada@udea.edu.co 
homosexuales como a aquellos que manifiestan que sus encuentros homoeróticos son solo casuales, nombrándose como bisexuales y aún como heterosexuales. Se estima que esta población tiene una prevalencia de la infección que oscila entre el 5 y el 40\% en América Latina (Estrada \& Vargas, 2011) y que contribuye en gran medida al aumento dramático de las cifras. La situación es aún más grave cuando se multiplica cada caso notificado por otros que aún no han sido diagnosticados. El primer caso de infección por VIH/SIDA reportado en Colombia fue en 1983 y hasta el 2015 se habían notificado un total de 124.868 casos de infección por VIH/SIDA y/o muerte (Cifras del VIH en Colombia, 2016, Diciembre 1), lo cual indica que en el país el $2,5 \%$ de la población vive con VIH. Sin embargo, existe un subregistro de la enfermedad, estimándose que la prevalencia del VIH es mucho más alta (Colombia, Ministerio de Salud y Protección Social \& UNFPA, 2012). Solo en el año 2015 se reportaron 11.414 casos con VIH/SIDA, de los cuales 8.666 fueron reportes por muerte, $76,6 \%$ fueron hombres y 23,2\%, mujeres (Cifras del VIH en Colombia, 2016, Diciembre 1). Del total de casos registrados hasta el 2010, $27.906(35,3 \%)$ no tenían identificado un mecanismo probable de transmisión (Colombia, Ministerio de Salud y Protección Social \& UNFPA, 2012). Para el 2017, la mayoría de infectados eran hombres $(79,9 \%)$ (Colombia, Instituto Nacional de Salud [INS], 2017) que temen revelar sus conductas sexuales a causa del estigma que, en ocasiones, acompaña al diagnóstico, y que se materializan en leyes discriminatorias y falta de accesos a servicios de prevención (Departamento de Salud y Servicios Humanos de EE. UU., 2016, Julio 8).

Antioquia, por su parte, fue el segundo departamento del país en notificación de casos para mediados de 2016, con un reporte de 857 casos de personas infectadas con VIH, 103 casos con SIDA y 24 muertes por SIDA (INS, 2016). Para esta misma fecha, se habían reportado 6.797 nuevos casos de VIH/SIDA y muerte por SIDA en todo el país (INS, 2016). Y en Medellín, para el año 2010, la prevalencia era del 9,1\% (Colombia, Ministerio de Salud y Protección Social \& UNFPA, 2011a).

En los años 80 la notificación de casos nuevos por año no sobrepasó los 24, mientras que en los años 90 la notificación creció exponencialmente, pasando los 300 casos en algunos años (Programa Conjunto de las Naciones Unidas sobre el VIH/SIDA, 1999). Para el año 2000, se evidenció un incremento en el reporte de casos clasificados como VIH (Colombia, Ministerio de Salud y Protección Social, 2013), tendencia que sigue presente en los últimos años: en el año 2013 se reportaron 8.208 casos (Colombia, Ministerio de Salud y Protección Social, 2016).

Las respuestas institucionales ante la magnitud del fenómeno han tenido como estrategia desde la represión del comportamiento, como la propuesta tradicional de los sectores religiosos que predican la abstinencia como forma de evitar el contagio de infecciones de transmisión sexual y una vía para alcanzar la salvación espiritual, hasta la educación masiva en los medios de comunicación, liderada por organismos convencidos de que la información permitiría a los grupos exponerse menos al riesgo; tales campañas, sin embargo, han demostrado que sirven para instalar el tema del SIDA y su prevención en la población general, pero no para modificar los comportamientos de riesgo (Cáceres, 2003).

Unido a los reportes epidemiológicos y por la complejidad del fenómeno, es importante pensar en los escenarios que influyen en el desarrollo de la enfermedad. En algunas culturas y en momentos históricos con circunstancias específicas, la condición homosexual ha sido señalada como anormal, perversa o pecaminosa, llevando al rechazo de este grupo. Según Barrientos y Cárdenas (2013), "las personas que forman parte de grupos minoritarios están expuestas a estresores sociales específicos: prejuicio, expectativas de rechazo, huida y ocultamiento" (p. 8). Como consecuencia, los HSH viven gran parte de sus procesos vitales en la clandestinidad, lo que puede influir en su capacidad para decidir, desde la autonomía, sobre sus comportamientos de riesgo para adquirir el virus del SIDA (Díaz Murillo, 2002), a partir de un contexto marginado en el que las posibilidades de elegir son restringidas. En Latinoamérica, tales contextos culturales tienen similitud y podrían explicar los entornos en los cuales las personas homosexuales viven su afectividad, experimentando muchos de ellos situaciones extremas de rechazo, lo que provoca sufrimiento psíquico y afectaciones en la forma como se negocian las prácticas de protección en los encuentros sexuales (GranadosCosme, Torres-Cruz \& Delgado-Sánchez, 2009). Investigaciones como la de Salazar et al. (2006) han encontrado que la fobia a la homosexualidad en condiciones de roles de género hegemónicas hace que la población homosexual interiorice prácticas de riesgo y disminuya las conductas de prevención, pese a tener conocimiento sobre la enfermedad. En Colombia, los colectivos de derechos humanos y activistas de la comunidad de lesbianas, gay, bisexuales y transexuales han denunciado el abuso policial, las violaciones en cárceles, los asesinatos por odio y otras formas de discriminación como maneras de violencia dirigidas hacia toda la comunidad homosexual (Lemaitre Ripoll, 2009). 
En Medellín, en la década de los 90 se desarrolló una investigación microetnográfica que intentaba comprender el significado de algunas de las vivencias que, frente a la epidemia del VIH/SIDA, experimentaban los grupos expuestos a un mayor riesgo de infección o enfermedad, las cuales pudieran explicar el éxito o el fracaso de los programas de intervención en la región (Seccional de Salud de Antioquia, 1993), encontrando que, aunque los participantes tenían un nivel aceptable de información frente al riesgo de sus comportamientos en relación con el VIH, seguían manteniendo tales conductas (Posada \& GómezArias, 2007). Hasta entonces los estudios en general no se habían detenido profundamente en el significado del riesgo para los grupos expuestos, como los HSH (Pisani, Brown, Saidel, Rehle \& Caraël, 1998), lo que llevó a los investigadores y autores de este artículo a plantearse una nueva investigación y preguntarse por qué persistían los comportamientos riesgosos en dicha población, aun teniendo información suficiente sobre la enfermedad.

El objetivo general de esta investigación fue elaborar una teoría consecuente con los significados de riesgo construidos por los HSH, entendiendo, así, su comportamiento y los elementos involucrados al momento de tomar decisiones respecto del riesgo frente al VIH/SIDA. Las conclusiones de este abordaje permiten asumir otros referentes para proponer estrategias de prevención que involucren el desarrollo social de otras actitudes que estén fundamentadas en la comprensión, a partir del ejercicio de escuchar y desmitificar a esta población.

\section{Método}

Se usó un enfoque cualitativo como soporte epistemológico. En este enfoque la indagación cualitativa tiene la pretensión de "generar conocimientos acerca de acontecimientos y procesos sociales al determinar lo que significan para las personas, explorar y documentar cómo interactúan las personas entre sí y cómo interpretan el mundo que los rodea e interactúan con él" (Ulin, Robinson \& Tolley, 2005/2006, p. 22). Se optó por el abordaje cualitativo debido al interés por comprender los significados relacionados con el riesgo y la vivencia de la sexualidad.

La teoría fundamentada (TF) como método de investigación se hizo pertinente para el abordaje de la realidad propuesta en esta investigación, ya que ella parte del interaccionismo simbólico, que intenta comprender la relación entre los comportamientos y los significados, planteando que los seres humanos son hacedores de su universo simbólico (Stryker, 1987), en el que crean y recrean significados para sus acciones (Blumer, 1969/1982). Strauss y Corbin (2002) denominan TF a la teoría derivada de datos recopilados de manera sistemática y analizados por medio de un proceso de investigación, en la que la recolección y el análisis de la información transcurren paralelamente.

\section{Participantes}

La estrategia de selección fue el muestreo teórico, consistente en una escogencia intencional de varios casos que pueden compararse y contrastarse. Estos casos se eligen por su posible relevancia para el campo teórico que se pretendió estudiar (Goetz \& LeCompte, 1984/1988).

Para determinar las características de los sujetos a seleccionar se identificaron informantes clave, tratándose de sujetos que cumplieran con estos criterios: relaciones homoeróticas, sexo masculino, mayores de 18 años, y con conocimientos básicos acerca del VIH/SIDA, como nociones elementales sobre infección, formas de prevención y de contagio, para así tratar de responder mejor a la pregunta sobre los significados construidos alrededor del riesgo, aun en presencia de información suficiente para prevenirse, pues, como lo sugerían los estudios previos, tener información no es condición suficiente para adoptar comportamientos preventivos frente al VIH.

Para contactar a los sujetos se usó el sistema voz a voz y se visitaron grupos organizados de la ciudad y sitios de reunión para socializar la invitación y seleccionar a los participantes; algunos de ellos hacían trabajo voluntario en algunas de las organizaciones. En la Tabla 1 se presentan algunos de los datos de los 19 hombres participantes del estudio. La recolección se detuvo cuando se encontró saturación teórica, concepto que, siguiendo a Strauss y Corbin (2002), apareció cuando los nuevos datos no aportaban elementos para alimentar las categorías de análisis. 
Tabla 1

Información de los Participantes del Estudio

\begin{tabular}{cccccc}
\hline Informante número & Edad & Autodefinición sexual & Escolaridad & Ocupación & Estado civil \\
\hline 1 & 32 & Homosexual & Universitaria & Bacteriólogo & Soltero \\
2 & 19 & Homosexual & Universitaria & Enfermero & Soltero \\
3 & 42 & Homosexual & Universitaria & Docente & Soltero \\
4 & 35 & Homosexual & $11^{\circ}$ grado & Desempleado & Soltero \\
5 & 42 & Homosexual & $11^{\circ}$ grado & Estilista & Soltero \\
6 & 39 & Homosexual & Tecnología & Estilista & Soltero \\
7 & 34 & Homosexual & Tecnología & Deportista & Soltero \\
8 & 28 & Homosexual & Tecnología & Operario & Soltero \\
9 & 46 & Homosexual & Universitaria & Activista gay & Soltero \\
10 & 20 & Homosexual & Tecnología & Estudiante & Soltero \\
11 & 44 & Bisexual & Universitaria & Terapeuta & Separado \\
12 & 27 & Homosexual & Técnico & Pensionado & Soltero \\
13 & 25 & Homosexual & Universitaria & Estudiante & Soltero \\
14 & 35 & Homosexual & Universitaria & Ingeniero & Soltero \\
15 & 27 & Homosexual & Universitaria & Docente & Soltero \\
16 & 23 & Homosexual & Universitaria & Abogado & Soltero \\
17 & 48 & Homosexual & $11^{\circ}$ grado & Desempleado & Soltero \\
18 & 22 & Homosexual & Universitaria & Jefe de área & Soltero \\
19 & 29 & Homosexual & Tecnología & Diseñador & Casado \\
\hline
\end{tabular}

\section{Instrumento}

Se usaron guiones de entrevista semiestructurada, que indagaban por los conocimientos previos en VIH, las interacciones con la familia, los amigos, la pareja, los comportamientos de riesgo y sus significados, tanto en el ámbito de la pareja y del individuo como del grupo, así como los comportamientos de prevención.

\section{Procedimiento}

Para el desarrollo de la investigación se tuvieron en cuenta los criterios éticos establecidos por la Declaración de Helsinki (Asociación Médica Mundial, 2013/2017) en lo concerniente al respeto por la condición de sujeto de todos los entrevistados. Se tuvo en cuenta el cuidado de su dignidad, seguridad, confiabilidad derecho a la participación y retiro voluntario. En cuanto al ámbito local, también se cumplieron los criterios de la Resolución 8430 de 1993 del Ministerio de Salud en Colombia, que, de forma similar a los criterios internacionales, regula las investigaciones desarrolladas con seres humanos en el país, invocando consideraciones para evitar el daño y proteger a los participantes. Al cumplir con estas condiciones, el comité de ética de la investigación en la Universidad de Antioquia otorgó los avales éticos para su desarrollo. Todos los participantes firmaron un consentimiento informado, en el que se estipulaban las condiciones para realizar la entrevista, la confidencialidad, el anonimato y la posibilidad de retirarse en el momento que así se deseara.

Las entrevistas fueron realizadas por la investigadora principal durante los años 2004 y 2005 y tuvieron una duración promedio de una hora. Estas se realizaban cuando y donde el participante designara. Cada una de ellas fue grabada y transcrita por una auxiliar técnica con un compromiso de confidencialidad.

\section{Análisis de la Información}

En la TF pueden definirse tres momentos de análisis, en el que se codifican los datos, es decir, se fragmentan, conceptualizan e integran para formar teorías (Strauss \& Corbin, 2002). El primer momento es denominado codificación abierta, consistente en un momento explicativo en el que los fenómenos interaccionan entre sí y del que surgen categorías descriptivas. En esta investigación surgieron 14 categorías de este tipo, que remitían a las nociones de riesgo, pareja, familia, homosexualidad, VIH y otras que fueron configurando un primer acercamiento a los resultados. Un segundo momento es llamado codificación axial: 
en este se reagrupan los datos fracturados de la codificación abierta, relacionando categorías y subcategorías analíticas que explican las condiciones y consecuencias del fenómeno. Este momento se tradujo en la reducción de las categorías anteriores a cinco de tipo analítico que empezaron a esbozar las relaciones y configuraciones de las categorías finales. En el tercer momento, la codificación selectiva, se integran y refinan las categorías analíticas, llegando a las interpretativas, para luego formar un esquema teórico mayor que da lugar a la teoría propiamente dicha. En este momento final se definieron cinco categorías para la construcción de la TF, que daban cuenta de los contextos en los cuales se relacionan y viven las personas HSH, los antecedentes para que los fenómenos de comportamientos de riesgo se presentaran y, por último, los factores que como condicionantes refuerzan la presencia de tales comportamientos, como la búsqueda del afecto en los encuentros eróticos con el otro y la construcción de una noción de autonomía en la estrechez de las interacciones entre HSH en la ciudad de Medellín.

\section{Resultados y Discusión}

Para comprender los resultados se presenta un esquema, en el que se encontraron dos categorías que fueron el eje central de la TF, permitiendo responder a la pregunta planteada (ver Figura 1).

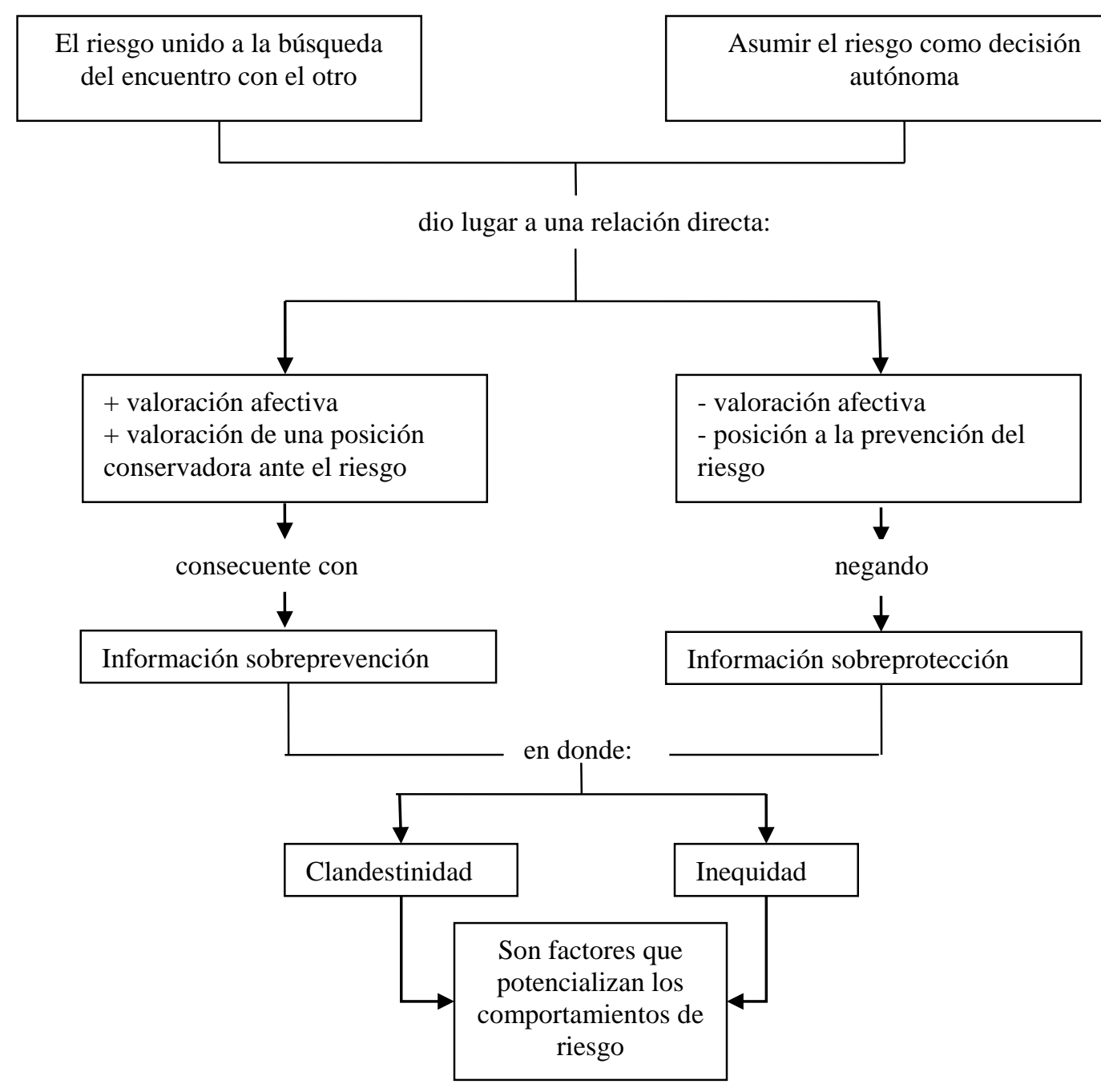

Figura 1. Categorías centrales para la $\mathrm{TF}$ de esta investigación y aquellas que potencializan la ocurrencia de los comportamientos de riesgo. 


\section{El Riesgo Unido a la Búsqueda del Encuentro con el Otro}

Uno de los hallazgos principales de la investigación fue observar que el riesgo se une a los significados que hacen parte del mundo afectivo de los HSH, pues en sus relatos no era posible encontrar el amor sin asumir una cuota de riesgo. En este punto es preciso señalar que el riesgo estaba relacionado en los discursos de los participantes en dos vías: (a) por el riesgo afectivo que involucra la relación amorosa con otra persona y (b) por el riesgo biológico que implica la disminución de prácticas de protección, como el uso de condón, para mantenerse en una relación a la que se le atribuye un gran valor en medio de la carencia. De esta forma, se pueden comprender las prácticas de riesgo por el significado atribuido al amor, que se asume como no inmune, no controlable. Esta relación entre prácticas y significados se presenta de forma coherente con los postulados del interaccionismo simbólico, que brinda los elementos teóricos para comprender los comportamientos a partir de los significados que de ellos se construyen en la interacción simbólica entre las personas.

La búsqueda de afecto se liga directamente con las nociones de apego y afecto, pues en la vivencia de la afectividad de los HSH se encontró una búsqueda constante de personas que representaran la posibilidad de la vivencia afectiva, originada en la ausencia de vinculaciones fuertes basadas en el respeto y la asunción de ellos en una categoría digna y que humaniza, tanto por parte de los otros como de ellos mismos.

El riesgo, entonces, como búsqueda constante, se manifiesta como un "pago" que debe hacerse en nombre del amor, esquivo en la mayoría de las historias de vida de los HSH, quienes, por su condición sexual, se ven sometidos a la exclusión de forma temprana, incluso por su misma familia. En este sentido, Bowlby (1988/1989) destaca la importancia que tienen los vínculos de apego establecidos con los padres durante la infancia para el desarrollo de posteriores relaciones afectivas.

Unida a la precariedad afectiva, manifiesta en las entrevistas, se encontró la vinculación de la clandestinidad como un factor que aporta condiciones que hacen difícil la adopción de conductas de prevención del VIH o cualquiera otra infección de transmisión sexual (ITS) y que, además, crea un escenario donde el intercambio afectivo no tiene un lugar propicio para su desarrollo. "Muchos de los espacios en los cuales los hombres tienen relaciones homosexuales clandestinamente (por ejemplo, calles, parques, automóviles, cines, saunas) harían muy difícil la incorporación del condón, en tanto otros riesgos estarían presentes; además, el actuar con rapidez sería visto como necesario" (Guajardo, 2002, p. 66). Ello se puede afirmar incluso teniendo en cuenta que existen lugares para el intercambio sexual y afectivo para los HSH, pero estos lugares todavía están sometidos al señalamiento y la clandestinidad en muchos casos, por el lugar de lo prohibido o pecaminoso con lo que aún se asocia a la homosexualidad. Así lo relataba uno de los entrevistados:

...Hay mucha gente que yo conozco, gente que al final no tiene un sitio, un lugar donde estar con su pareja o con quien vaya a estar, entonces, tiene que recurrir a un escondite, a cualquier sitio, a las carreras, y en esas carreras esta gente no utiliza ningún tipo de prevención, solamente vamos a lo que vinimos y punto, entonces, pienso que desde ahí la gente no se cuida tanto o simplemente porque hacen falta medios o porque eran tan señalados como personas, sentían la necesidad de tener sexo y lo terminaban teniendo en cualquier lado (Entrevista 14, página 2).

Asimismo, en el escenario cultural la clandestinidad se asocia con lo que no es aceptado, con lo inmoral y la ilegalidad y de allí que un hombre que se halle teniendo sus encuentros de pareja en estas condiciones también adopta para sí mismo esta calidad de sentirse ajeno, por fuera de lo que es lícito y, consecuentemente, menos valorado socialmente, lo que podría explicar que los mismos HSH adopten conductas de riesgo que son contrarias al mantenimiento de una vida saludable y que los acerca cada vez más a la muerte como horizonte próximo, muerte asociada a la condición homosexual y al VIH (García Abreu, Noguer \& Cowgill, 2003/2004), lo que confirma la asociación de los significados de subvaloración por ser homosexual con las prácticas de riesgo. Uno de los entrevistados mencionaba:

En la medida en que yo tengo que esconderme para hacer las cosas... me vuelvo más vulnerable ... porque, entonces, tengo menos tiempo... estoy a la carrera... estoy llegando a lugares que no son sanos... estoy llegando a lugares de mucho más riesgo... la clandestinidad siempre va a llevar a un riesgo mucho más alto... y con lo del VIH mucho más... porque entonces es... todo lo que tiene que ver... con la autoestima y la identidad... que tiene que ver con que... tú estás escondiéndote y tienes que estar jugando doble papel... etc ...etc. [...] Entonces, eso aumenta el riesgo. (Entrevista 3, página 16).

Aunado al hecho de tener que vivir en esta clandestinidad, se ha encontrado que la vida de muchos de los HSH entrevistados está marcada por una serie de carencias afectivas con las figuras que, desde sus etapas tempranas, representan la posibilidad de amor, protección y compañía, fenómeno que puede entenderse en un escenario donde los valores de la heterosexualidad y masculinidad priman, lo que podría llegar a explicar que la discriminación y la homofobia se manifiesten aun en las figuras familiares, las que, a partir de su 
inserción social, justifiquen la agresión hacia aquel que consideran fuera de la norma: al hombre homosexual. Si bien esta afirmación pareciera confirmar un modelo en el que los hombres homosexuales son educados en una cultura heterosexista, ello fue lo relatado por los entrevistados y en TF la realidad es lo que los actores sociales dicen que es. Como se menciona en Barrientos y Cárdenas (2013), "si el que violenta el rol de género es hombre, esta trasgresión es vista como más grave que en el caso de las mujeres" (p. 6). Para muchos HSH esta situación permite que, desde su adolescencia y en la adultez, la pareja se constituya como la única probabilidad de obtener un vínculo afectivo; de allí su sobrevaloración y la entrega absoluta a ese otro cada vez que se hallan ante la posibilidad de amar. Este hecho permitiría explicar, a su vez, la necesidad constante de estar en pareja y la búsqueda que ello significa cuando las rupturas son constantes en las situaciones atípicas en las que se vive la relación homosexual.

Respecto de los roles de género, los hombres suelen tener muchas más parejas que las mujeres (GarcíaSánchez, 2004), también los homosexuales, lo que pareciera reafirmar los mandatos patriarcales aun para los hombres que prefieren a otros hombres como compañeros. El lugar adjudicado a la pareja, el consecuente miedo a encontrarse solo y las condiciones de clandestinidad en las que se desarrollan sus encuentros eróticos y afectivos permiten que el riesgo se una a la búsqueda y vivencia de la pareja. En este punto la pareja representa la única salida posible a la carencia afectiva y de allí, las prácticas de riesgo en el intento de mantenerla. Uno de los entrevistados afirmaba:

El amor surge, adquiere una importancia tal que la gente aun así lo tiene, con un riesgo en su vida, porque es que cuando uno es homosexual en una sociedad como esta, no solamente está poniendo en riesgo su vida por el VIH desde la parte infectológica, sino también pues porque a los homosexuales los matan por ser homosexuales, entonces, yo pienso que si uno sabe que está siendo homosexual y que si es probable que por eso lo maten, pues no creo que tampoco se vaya a preocupar mucho por tomar una decisión de VIH sí o VIH no, pues igual está en juego la vida. (Entrevista 16, página 4).

Sumado a esto, los participantes no solo admitieron el riesgo en cuanto a los encuentros que tenga la pareja durante la permanencia del vínculo como tal, sino que también asumen el riesgo después de conocer las historias sexuales anteriores de sus compañeros, las que pudieron estar también caracterizadas por encuentros riesgosos, implicando que el diagnóstico de cualquiera ITS aún no sea conocido. Ello puede unirse al efecto en la percepción de la población respecto de la eficacia de las terapias antirretrovirales para el VIH, produciendo una disminución de las conductas de prevención, amparada en la esperanza de protección y tratamiento que ofrecen estos medicamentos — lo que ya ha sido abordado por algunos expertos (Aragonés López, Campos Díaz, Sánchez Valdés \& Pérez Ávila, 2007; Juarez-Vúlchez \& Pozo, 2010)—o a la profilaxis preexposición (PrEP), consistente en la toma de medicamentos específicos para el VIH por parte de personas que no tienen el virus pero que corren el riesgo de contraerlo. De acuerdo con el Centro de Control y Prevención de Enfermedades de Estados Unidos, la PrEP reduce en más de un 90\% el riesgo de contraer la enfermedad a través de las relaciones sexuales (Departamento de Salud y Servicios Humanos de EE. UU., 2019, Mayo 21). Tanto la eficacia de las terapias antirretrovirales como la PrEP brindan elementos para que la prevención deba ser revalorada, en tanto el VIH pareciera ir perdiendo su significación de letalidad.

Esta posición frente al riesgo de contraer el VIH se une a la manera como aman los HSH:

Yo soy una persona muy libre y no puedo estar metida todo el tiempo y los homosexuales somos más intensos en las relaciones... somos muy intensos. Tanto es que los homosexuales promiscúan sus relaciones, es porque sus relaciones duran muy poco. A unos las relaciones les duran un mes. Uno al mes ya cree que lleva casi uno o dos años... Porque las relaciones se viven intensamente. Ahora se conocen... a la hora ya están teniendo relaciones y a las dos horas ya somos pareja. Así son de intensas las relaciones (Entrevista 1, páginas 10-11).

En varias ocasiones los entrevistados hicieron énfasis sobre la existencia del amor en sus encuentros de pareja, pero, también, admitían cómo el contexto y la historia social de la homosexualidad se constituían en un elemento que empujaba a vivir rápidamente cualquier oportunidad de relación con uno de sus pares. Y así como se vive de forma intensa el inicio y la consolidación de la pareja, también llega pronto el desencuentro, la ruptura y el inicio de una nueva relación, permitiendo que un HSH pueda llegar a tener en un solo año varios compañeros sentimentales y sexuales. Ello contribuye a que el riesgo de contraer VIH o alguna ITS es mayor, pues, al estar vinculados por medio del amor o de su promesa, se hacen compromisos y demostraciones de fidelidad, que incluyen no utilizar preservativo. Es decir, el riesgo se incrementa por la vinculación con el amor y por las prácticas que se imaginan atadas a la pareja formal: la confianza absoluta y la fidelidad.

Esta poca duración en las relaciones de pareja o la búsqueda constante de nuevos compañeros pareciera responder, según algunos investigadores, a: (a) una necesidad infinita, y no resuelta, de estar acompañados; 
(b) una subvaloración de sí, en tanto se es un hombre con conductas homosexuales que no responde al rol exigido socialmente (Salazar et al., 2006); (c) la disolución de la pareja, que se presenta más frecuentemente en relaciones homosexuales, por la carga y poco soporte social que crean contextos poco favorables para la perdurabilidad de esta relaciones de pareja (investigaciones como la de Gallego Villa y Barreiro de Motta, 2010, establecen que no hay diferencias estadísticamente significativas en la perdurabilidad de las relaciones entre hombres gay y mujeres lesbianas, aunque en algunos estudios se encuentra información sobre la mayor duración de las relaciones en ellas, probablemente asociada al rol de género, que liga la estabilidad con lo femenino); y (d) un estereotipo acerca de la inestabilidad y violencia que pareciera acompañar los imaginarios sobre las relaciones entre hombres homosexuales (García et al., 2017), lo que acabó por homologar la conducta homosexual con la promiscuidad, como una combinación inherente a la cotidianidad de estas personas, que se oponía a la monogamia impuesta por los modelos tradicionales y que, por supuesto, terminaba siendo castigada por enfermedades como el SIDA, según los imaginarios sociales construidos (Muñoz, 1996). Debe añadirse que ninguna de estas explicaciones es suficiente por sí misma para explicar la corta duración que expresan los HSH en sus relaciones de pareja, pero sí pueden dar algunas pistas de lo que ocurre con este fenómeno, íntimamente relacionado con el contexto y con el tipo de interacciones que pueden establecer estas personas en medios que los restringen socialmente.

Este modelo que se sigue como estrategia para encontrar y mantener el afecto niega la información que se tiene en cuanto a riesgo y prevención. Sin embargo, no solo debe tenerse en cuenta el riesgo de contraer el VIH, sino también el riesgo de sufrir emocionalmente, pues, así como una persona heterosexual ha vivido momentos en los que se ha sentido herido afectivamente, los HSH han expresado que el hecho de ser homosexuales hace que el sufrimiento se presente más por el número de veces que puede llegar a enamorarse. Un entrevistado expresaba:

Asumimos un papel de un enamorado (...) Porque uno piensa que el tener pareja es tan bueno que lo pone a uno a sufrir a la vez. Entonces, son contradicciones que uno aprende a conocer... no sé si comprendas lo que te estoy diciendo, pero... pero, el concepto, por lo general de nosotros, es que amar es tan bueno que... que uno sufre. Entonces, en el caso del homosexual se ve más el sufrimiento. Y se ve más la promiscuidad; es porque uno no alcanza a conocer nunca a la pareja que está compartiendo con uno. (Entrevista 7, páginas 12-13).

Además de esto, estudios realizados por psicólogos y psiquiatras han podido establecer que, unido al contexto social, hay estereotipos — modelos que se aprenden a partir del concepto que sobre el homosexual tiene la sociedad cuando señala y recrimina su conducta - relacionando la homosexualidad con la perversión, la promiscuidad y la satisfacción de instintos netamente sexuales. Los hombres que mantienen conductas homosexuales, asumiéndose o no públicamente como tales, se hallan ante un esquema de rechazo social que puede asumirse como natural a su condición, lo que facilita mantener los modelos públicos que el imaginario social ha construido y, de esta manera, también mantener las conductas de riesgo que los acompañan. Esta asociación confirma de nuevo la relación indisoluble entre lo que se piensa de sí mismo como homosexual, confirmando el estigma de los imaginarios sociales, y las consecuentes prácticas de riesgo, como énfasis del menosprecio público orientado hacia sí mismo. Esta asociación está en íntima consonancia con los postulados interaccionistas que guiaron la comprensión teórica en esta investigación.

En el lado opuesto de los prejuicios sociales, hay teorías, como las de Gómez-Beneyto (1978), que entendiendo por homosexualidad la capacidad para amar al propio sexo, subrayan el hecho homosexual como una capacidad positiva de cualquier ser humano, señalando que se trata de amor y no solo de sexo entre dos personas del mismo sexo. El amor para este psicólogo-psiquiatra incluye la relación sexual y la interpersonal y también hace referencia a un ámbito más creador y positivo.

\section{Asumir el Riesgo como Decisión Autónoma}

En el marco de la investigación, el concepto de autonomía surgió como una construcción particular de los HSH, desde su contexto y su devenir histórico. Estos consideran que un individuo puede decidir y acertar en su orientación sexual:

Yo pienso que cada persona decide lo que va a hacer con su vida y se me podría decir que la sociedad muchas veces no nos ofrece alternativas de vida, pero vemos casos en que las personas en las peores condiciones salen adelante, porque toman la decisión de salir adelante. (Entrevista 11, página 3).

Estudios como los de Lluch Canut (1999) vinculan la autonomía con una medida positiva para establecer la salud mental; un sujeto percibe que es capaz de guiarse por normas que le son propias, sin negar las exigencias del ambiente. En este sentido, la autonomía sería una negociación entre los deseos propios y la 
voluntad de los otros. En el ámbito de la sexualidad, algunos estudios han podido demostrar la relación entre la autonomía como componente de la salud mental y los comportamientos de autoprotección, lo que no solamente aplica a las personas que tienen prácticas homosexuales, sino a todas aquellas que han sido expuestas a condiciones de vulnerabilidad debido a su rol social, como es el caso de las mujeres (Canaval et al., 2005). En algunos de estos casos se ha encontrado que, sumados a la alta autoestima, existen otros factores de protección, como la independencia económica y familiar (Collazos, Echeverry, Molina, Canaval \& Valencia, 2005). Ello demuestra que la vulnerabilidad y la autoestima parecieran tener profundas relaciones en cualquier población sometida al aislamiento, en la que una estima alta pareciera conducir a la protección propia. En esta relación se confirma de nuevo la visión interaccionista de la realidad social, donde un significado de alta valoración de sí conduce a prácticas consecuentes con la protección y el valor del cuerpo y la salud.

Por otro lado, Eric Fromm (1955/1964) presenta el concepto de individualidad, señalando que, aunque el hombre tiene arraigados unos deseos orientados a decidir por sí mismo en libertad, es precisamente su necesidad de agruparse la que finalmente determina su conducta, es decir, que lo que es esperado en un futuro miembro de un grupo y que lo haría ser aceptado en este es mucho más importante que su necesidad de libertad y autonomía. Desde la teoría de la identidad social, el actor social define su identidad seleccionando y jerarquizando las fronteras de sí mismo con aquellos elementos que simbólicamente lo definen en el proceso de interacción. Es la identidad la que origina el autorreconocimiento, la autorreflexión e, incluso, la autonomía como expresión de la subjetividad, que se pone en juego y tensión en la interacción social y en las decisiones en las que se manifiesta tal necesidad de mantenerse reconocido como miembro de un grupo (Giménez, 1996).

Esta tensión representó para el grupo de HSH la disyuntiva entre lo que sus familias y el medio social esperan de ellos como varones y lo que comienzan a descubrir en su adolescencia como hombres que se sienten atraídos erótica y afectivamente por otros hombres. Esto puede explicar el hecho de que muchos hombres asuman su homosexualidad como circunstancial y no como parte esencial de sus vidas, respondiendo públicamente como heterosexuales y manteniendo en la clandestinidad sus encuentros y parejas homoeróticas (Ardila, 1998). Sin embargo, se ha planteado que el acompañamiento y el afecto brindado de forma constante por parte de las familias, aún después de conocer las preferencias sexuales de uno de sus miembros, se reconoce como punto de partida para la elección de una posición, generalmente la de protección en sus comportamientos frente al VIH. Se entiende que la autonomía así tratada corresponde a un elemento presente a la hora de elegir y no a una decisión en sí. La relación así presentada entre la autonomía y las decisiones de protección y riesgo enfatiza la necesidad de reforzar tal componente de la salud mental desde edades tempranas y más específicamente en adolescentes de cualquier preferencia sexual que inician de forma precoz su exposición al riesgo. En ellos se ha podido validar la hipótesis de que a mayor autoestima mayor es el comportamiento de protección, aun en contextos latinoamericanos y patriarcales donde las condiciones culturales pueden ser adversas, por el rechazo a la presencia de rasgos homosexuales en los varones (Lozano Santa \& Talexio Paredes, 2011). Es precisamente en estos medios donde se hace necesaria tal intervención, para procurar modificar los significados en torno a la masculinidad y la homosexualidad desde edades tempranas, pues la homofobia, el miedo y el sufrimiento psíquico que genera este medio, que solo aprueba la supremacía de la heterosexualidad, se ha relacionado con la baja negociación y la presencia significativa de situaciones de riesgo de contraer el VIH en la población homosexual (Granados-Cosme et al., 2009).

Cuando los entrevistados fueron indagados por su autonomía y por la forma como hacen elecciones frente al riesgo, estos expresaron que las decisiones que han tomado de forma consistente con sus expectativas y con sus necesidades correspondían a decisiones autónomas, en la vía de la satisfacción de la necesidad aceptada de compañía o en la vía del compromiso ético con la salud o la fidelidad. Así lo manifestaba un entrevistado:

... el riesgo de tener relaciones sexuales sin protección es un riesgo en el cual tu eres responsable y nadie más, nadie más. Ahora ¿qué tiene que ver ese riesgo? ¿qué tiene que ver esa decisión? Tiene que ver con la autonomía que hayas tenido, con la formación que hayas tenido, con el contexto social en el que te has levantado, con el tipo de familia que te ha tocado, etc., etc. Creo que es con las herramientas y las habilidades para enfrentar la vida y para tener una actitud frente a la vida. ¿Cuál sería la diferencia? (...) que unos hemos tenido más oportunidades que otros y, entonces, las decisiones van a ser más acertadas o menos acertadas. (Entrevista 3, página 8).

Con respecto a la decisión que toman los HSH frente al amor, se ha manifestado que la afectividad positiva promueve una aceptación de la condición homoerótica y previene la toma de decisiones contrarias al deseo de los sujetos, que vienen impuestas socialmente como modelos establecidos que se asumen por la profunda falta de afectividad, por encima de la satisfacción de los individuos (Eribon, 2000). Esta afectividad 
positiva, que se establece desde los primeros vínculos, promueve que paulatinamente se vaya adquiriendo una autonomía cada vez mayor. En palabras de Bowlby (1973/1985), "cuando esta se presenta paralelamente a la transmisión de modelos que resulten tolerablemente válidos y además que puedan ser cuestionados y modificados, permitirá un vínculo amoroso sano desde la niñez que, unido a la autonomía, propiciará elecciones que no impliquen un riesgo mayor de hacerse daño" (p. 347). En este sentido, cuando a uno de los entrevistados se le indagó por la relación que tienen las vivencias familiares con lo que él considera de sí mismo, expresó:

Mi mamá confía plenamente en nosotros. Mi papá es una persona que también, eso sí que menos, no se mete en la vida sexual de nosotros. Ha sido una persona muy respetuosa. Desde que ellos saben que yo soy homosexual, no se han metido como a instigarme o a hacerme sentir diferente, no... En mi casa... Y mis hermanos... pues no... me tratan normal. (Entrevista 1, página 4).

Debe considerarse que la autonomía es fruto de un proceso y que a medida que un HSH va desarrollando habilidades críticas sobre el riesgo, su responsabilidad también aumenta con relación a las opciones que toma en la consecución de pareja. Dichas habilidades pueden adquirirse brindando información sobre VIH y otras ITS por medio de campañas y con la implementación de intervenciones que promuevan la equidad, los derechos y la promoción de condiciones en las que el ser humano puede desarrollar su sexualidad. Sin embargo, en una de las entrevistas el interlocutor, aunque respalda el uso de la información que se brinda en estas campañas de prevención masivas, manifiesta que a la hora de elegir son otros los factores que toman fuerza para el balance entre pérdidas y ganancias y la posterior decisión:

Esa información sirve todo el tiempo, si va a haber un encuentro fugaz, si tú tienes esa información tienes que asumir si vas o no vas a tomar ese riesgo de esa relación fugaz, pero es que la información es... lo que más claro debe quedar entre toda la información sobre el VIH y las ITS es saber tomar una decisión y que la decisión sea tuya, saber con quién, cómo, cuándo y por qué. (Entrevista 19, página 7 ).

La brecha entre la información y el acto permite pensar en la propuesta teórica de Benasayag (1996), que relaciona la interiorización de contenidos con la dimensión afectiva de estos para los sujetos; en este caso, la información que se imparte respecto de la prevención del VIH (el uso de preservativo) correspondería a aquello que no se interioriza mientras que no se enlace con los significados afectivos y, de allí, con el desarrollo de un alto afecto hacia sí mismo. Por ello, es relevante hacer este tipo de investigaciones, pues se establece que es la presencia o ausencia de una afectividad positiva previa la que pareciera determinar el tipo de decisión tomada, en una profunda relación entre significados de la pareja, del afecto y del ser HSH con los comportamientos de riesgo o de protección frente al VIH.

Un ejemplo de lo anterior se reflejó en una entrevista, en la que el interlocutor optó por elegir el riesgo como consecuencia de la falta de respaldo familiar, lo que lo obligó a la clandestinidad. Este riesgo no fue tomado como una imposición sino como su opción, mientras no resolviera sus faltas afectivas. Sin embargo, más adelante, el mismo interlocutor relata cómo su vida ha cambiado y cómo ahora sus decisiones apuntan hacia otras direcciones. Expresa que este cambio se debe a la vivencia positiva de su afectividad y de su homosexualidad:

Bueno, yo creo que, con relación a la sexualidad misma, en este momento no corro tantos riesgos, entonces, mirándolo ahora, yo creo que eso tiene que ver con la manera como yo mismo he logrado entenderme, aceptarme y quererme, entonces, ya no quiero arriesgar lo que he obtenido, no quiero arriesgar ni mi relación, ni lo que estoy ahora construyendo realmente ni como me siento, entonces, ahora me cuido, me cuido y también me siento responsable de mí mismo y de mi pareja. (Entrevista 11, páginas 17-18).

Este tipo de relatos provee aportes fundamentales para pensar la relación autoestima-autocuidado, pues permite concluir que si una persona, independientemente de su vivencia de la sexualidad, está investida por afecto positivo y valora su bienestar, muy probablemente opte por comportamientos de autocuidado. Esta conclusión se vincula con la evidencia en esta investigación de que el aislamiento y la discriminación potencializan la opción de riesgo como camino válido para el relacionamiento con el otro.

Si la clave de la opción tomada por los HSH es el estado de su afectividad, es precisamente allí donde se hace pertinente y necesaria la intervención de las campañas que promueven conductas de protección frente al VIH/SIDA, es decir, que estas acciones deben estimular individual y socialmente una valoración de los homosexuales en términos de su condición digna de seres humanos, valoración que parta de ellos mismos y de otros grupos sociales. "Existe evidencia de que 'salir del closet', sumado a adecuados niveles de soporte social real y/o percibido (redes sociales disponibles), tiene efectos positivos en el ajuste psicológico y la autoestima, ya que proveería un modelo de rol homosexual (Teasdale \& Bradley-Engen, 2010) y una comunidad de referencia que valida la experiencia del individuo" (Barrientos \& Cárdenas, 2013, p. 8). Se trataría, entonces, de abordar como problema la inequidad y la clandestinidad a las que son sometidos bajo 
presión los HSH, creando el contexto para elegir el riesgo y exponer cómo el apoyo y la posibilidad de expresar afectos se puede convertir en un dispositivo de prevención que dispone a un individuo para su propio cuidado.

Es así, entonces, como el riesgo o el no riesgo se vincula con la ausencia o presencia del amor, según los relatos de los entrevistados, los que, en su inmersión cultural, relacionan el amor y la fidelidad como la mejor estrategia para evitar la infección, olvidando que es el sexo inseguro el comportamiento que los expone con mayor probabilidad al contagio de cualquier ITS o del VIH, postura que se acerca a los planteamientos generados por sectores conservadores e influyentes en las sociedades de América Latina y que se preservan aún hoy como mitos que son transmitidos y reproducidos con facilidad (Colombia, Ministerio de Salud y Protección Social \& UNFPA, 2011b).

Sin embargo, en palabras de los participantes, se debe sumar a toda esta trama un elemento adicional: que la vinculación con el amor explica la decisión, más no absuelve de la responsabilidad al que decide. Así lo expresa un entrevistado, quien, luego de su diagnóstico como seropositivo, ha dado la vuelta a su visión sobre la vida y sobre el papel que juega su afectividad en la toma de decisiones:

Yo creo que cada uno tiene la libertad de ser como cada uno quiere. Cada uno asume sus propios riesgos [...] Si yo acepto tener una relación sexual con una persona que no conozco, y que apenas la estoy conociendo, sin ninguna protección, es porque yo no me quiero. O sea, yo estoy asumiendo el riesgo de infectarme, no solamente de VIH, sino también de cualquier ITS. O sea que soy yo. Entonces, simplemente, si yo me quiero, yo me cuido.

Si yo no me quiero y quiero asumir ese riesgo... es porque no me quiero en sí. Entonces, porque... el mundo entero... en este momento, el mundo entero... sabe que hay una enfermedad, que es de transmisión sexual, que se llama VIH/SIDA y que le da a negros, blancos, feos, bonitos... los de cualquier raza. (Entrevista 4, páginas 15, 18).

En el fragmento anterior puede encontrarse una serie de pistas para pensar el problema del mantenimiento de conductas de riesgo y para proponer vías para intentar modificarlas. También vale la pena considerar que, según las palabras del entrevistado, la responsabilidad del contagio del VIH se focaliza en la conducta individual, pareciendo dejar de lado la responsabilidad social y el papel de la exclusión y el estigma en la adopción de estas conductas de riesgo. Sin embargo, debe resaltarse que el amor propio del que habla el entrevistado surge de la interacción con otros y del estigma o aceptación con el que es tratado. Es decir, se trata, entonces, de retomar las pistas que ofrecen los involucrados para el diseño y seguimiento de intervenciones, pero teniendo en cuenta que su propio discurso también está inmerso en una cultura estigmatizadora que los lleva a reproducir y encarnar el estigma originado en la interacción social como una huella internalizada que profundiza la culpa y la ansiedad y que permite, a su vez, la transgresión y el señalamiento de los otros (Chapman, 2000).

\section{Conclusiones}

Aunque la recolección de la información se hizo en los años 2004 y 2005, las autoras han seguido investigando el fenómeno en años posteriores, encontrando que los hallazgos siguen siendo pertinentes. Esta afirmación se sustenta en que el eje vertical de lo presentado en los resultados es la búsqueda de afecto en los encuentros eróticos entre HSH, su relación con la autonomía y, a partir de la mirada del interaccionismo simbólico, los significados que se atribuyen a tales comportamientos, aun constituyendo prácticas de riesgo. Si bien en la actualidad ha habido innovación en las prácticas retrovirales que permiten un mayor bienestar para los portadores del VIH, la condición de aislamiento y señalamiento hacia la población con prácticas homosexuales sigue estando vigente en los países latinoamericanos. Tal escenario constituye un marco simbólico particular para estudiar sus prácticas de riesgo.

La vivencia del riesgo en los participantes de contraer VIH y otras ITS se enmarca en la construcción social de los significados sobre su sexualidad y sobre el estigma asociado al ser homosexual. Ello tiene una profunda implicación para las decisiones sobre el riesgo, pues ellas se justifican en un contexto de obligatoria clandestinidad, por el rechazo que experimentan para sus encuentros afectivos.

Las relaciones homoeróticas siguen estimándose, aun por una gran cantidad de los HSH, como pecaminosas y anormales, lo que tiene profundos efectos en el reconocimiento y autoestima, lo cual puede explicar el mantenimiento de comportamientos de riesgo, aun habiendo información suficiente sobre la enfermedad.

La fusión de carencias afectivas y discriminación da pie a una sobrevaloración de los encuentros de pareja como único soporte afectivo de la vida adulta; el afecto allí vivido se equipara al reconocimiento de un ser humano que, en circunstancias similares, es valorado y legitimado por otro. 
En un marco restringido de posibilidades, los HSH eligen el riesgo como opción para vincularse con otro en la búsqueda de afectividad, la que califican como autónoma. Sin embargo, si el marco de referencia para las elecciones se amplía, desde la fortaleza afectiva vivida previamente y la aceptación de la propia homosexualidad, las decisiones tienden a la autoprotección como reconocimiento de la valoración de sí mismo.

Si bien la autonomía en los HSH corresponde a una negociación entre los deseos individuales y las exigencias del medio en el cual se desenvuelven, ella está mediada por la valoración de sí y por la exclusión y enjuiciamiento moral al que son sometidos los HSH. Tal exclusión aumenta la vulnerabilidad social de esta población, haciendo que la autonomía se viva en un contexto mucho más restringido.

Para construir una manera de pensar y actuar autónoma y consistente con la forma como aman y desean, los HSH han optado por alejarse de los esquemas tradicionales aceptados socialmente, constituyendo para el grupo normas propias que regulan la forma como establecen sus relaciones de pareja.

Las campañas de promoción de la salud y prevención del VIH deben comprender que el riesgo se incrementa y se valida por la aceptación social de esquemas únicos de conducta impuestos por modelos tradicionales y patriarcales, que censuran a los $\mathrm{HSH}$, restringiendo sus encuentros de pareja a la clandestinidad. Esto implica que la información por sí sola no es suficiente para generar cambios orientados a la prevención.

\section{Limitaciones del Estudio y Consideraciones Prácticas}

Debido al enfoque cualitativo del estudio y por el consecuente pequeño tamaño de la muestra, esta investigación ofrece resultados que pueden dar pistas sobre lo que ocurre con los grupos de HSH que viven en la ciudad de Medellín, sobre sus significados y prácticas de riesgo de contraer el VIH. Sin embargo, tales resultados no son generalizables a otras poblaciones.

En cuanto a las consideraciones prácticas, los hallazgos fueron presentados a varias organizaciones de la ciudad que realizan activismo político y que influyen en el diseño y desarrollo de políticas, planes y proyectos en torno a la población homosexual y las condiciones que favorecen el incremento de los casos de VIH/SIDA.

\section{Referencias}

Aragonés López, C., Campos Díaz, J. R., Sánchez Valdés, L. \& Pérez Ávila, L. J. (2007). Grupos de prevención del SIDA (GPSIDA): 15 años de trabajo sostenido en la prevención del VIH/sida. Revista Cubana de Medicina Tropical, 59, 261-269. Extraído de http://scielo.sld.cu/scielo.php?script=sci_arttext\&pid=S0375-07602007000300014\&lng=es\&tlng=es

Ardila, R. (1998). Homosexualidad y psicología. Bogotá, Colombia: Manual Moderno.

Asociación Médica Mundial (2013/2017). Declaración de Helsinki de la AMM - Principios éticos para las investigaciones médicas en seres humanos (traducción de la Declaración de 2013). Ferney-Voltaire, Francia: Autor. Extraído de https://www.wma.net/es/policiespost/declaracion-de-helsinki-de-la-amm-principios-eticos-para-las-investigaciones-medicas-en-seres-humanos/

Barrientos, J. \& Cárdenas, M. (2013). Homofobia y calidad de vida de gay y lesbianas: una mirada psicosocial. Psykhe, 22(1), 3-14. https://doi.org/10.7764/psykhe.22.1.553

Benasayag, M. (1996). Pensar la libertad: la decisión, el azar y la situación. Buenos Aires, Argentina: Nueva Visión.

Blumer, H. (1969/1982). El interaccionismo simbólico: perspectiva y método (P. Ridruejo Alonso, Trad.; Título original: Symbolic interactionism: Perspective and method). Barcelona, España: Hora.

Bowlby, J. (1973/1985). La separación afectiva (Título original:Separation: Anxiety and anger). Barcelona, España: Paidós Ibérica.

Bowlby, J. (1988/1989). Una base segura: aplicaciones clínicas de una teoría del apego (E. Mateo, Trad.; Título original: A secure base: Clinical applications of attachment theory). Barcelona, España: Paidós Ibérica.

Cáceres, C. F. (2003). La prevención del VIH/SIDA en América Latina y el Caribe. Washington, DC: Banco Interamericano de Desarrollo.

Canaval, G. E., Valencia, C. P., Forero, L., Guardela, N., Magaña, A. \& Vargas, Y. (2005). Factores protectores y de riesgo para VIH/SIDA en mujeres de Cali, Colombia. Ciencia y Enfermería, 11(2), 23-33. https://doi.org/10.4067/S0717-95532005000200005

Chapman, E. (2000). Conceptualisation of the body for people living with HIV: Issues of touch and contamination. Sociology of Health \& Illness, 22, 840-857. https://doi.org/10.1111/1467-9566.00233

Cifras del VIH en Colombia en el Día Mundial de Lucha contra el Sida (2016, Diciembre 1). Opinión \& Salud.com [Revista Digital]. Extraído de https://www.opinionysalud.com/cifras-del-vih-colombia-dia-mundial-lucha-sida/

Collazos, M. F., Echeverry, N., Molina, A. P., Canaval, G. E. \& Valencia, C. P. (2005). Riesgo de VIH/SIDA en la mujer: no es cuestión de clase. Colombia Médica, 36(3 Supl 2), 50-57. Extraído de http://colombiamedica.univalle.edu.co/index.php/comedica/article/view/376/380

Colombia, Instituto Nacional de Salud (2016). Boletín epidemiológico semanal: semana epidemiológica número 29 de 2016,17 julio-23 julio. Bogotá, Colombia: Autor, Dirección de Vigilancia y Análisis del Riesgo en Salud Pública. Extraído de https://www.ins.gov.co/buscadoreventos/BoletinEpidemiologico/2016\%20Bolet\%C3\%ADn\%20epidemiol\%C3\%B3gico\%20semana\%2029.pdf

Colombia, Instituto Nacional de Salud (2017). Informe de evento VIH/SIDA, Colombia, 2017. Bogotá, Colombia: Autor. Extraído de https://www.ins.gov.co/buscador-eventos/Informesdeevento/VIH-SIDA\%202017.pdf 
Colombia, Ministerio de Salud (1993). Resolución número 8430 de 1993, por la cual se establecen las normas científicas, técnicas y administrativas para la investigación en salud. Bogotá, Colombia: Autor. Extraído de https://www.minsalud.gov.co/sites/rid/Lists/BibliotecaDigital/RIDE/DE/DIJ/RESOLUCION-8430-DE-1993.PDF

Colombia, Ministerio de Salud y Protección Social (2013). Boletín Epidemiológico, situación del VIH/Sida, Colombia 2013. Bogotá, Colombia: Autor. Extraído de https://www.minsalud.gov.co/Documentos\%20y\%20Publicaciones/BOLETIN\%20EPIDEMIOLOGICO\%20VIH\%2019832012.pdf

Colombia, Ministerio de Salud y Protección Social (2016). Análisis de situación de salud (ASIS). Colombia, 2016. Bogotá, Colombia: Autor. Extraído de https://www.minsalud.gov.co/sites/rid/Lists/BibliotecaDigital/RIDE/VS/ED/PSP/asis-colombia-2016.pdf

Colombia, Ministerio de Salud y Protección Social \& Fondo de Población de las Naciones Unidas (2011a). Comportamiento sexual y prevalencia de VIH en hombres que tienen relaciones sexuales con hombres en siete ciudades de Colombia. Bogotá, Colombia: Autores. Extraído de https://www.minsalud.gov.co/salud/Documents/observatorio_vih/documentos/monitoreo_evaluacion/2_evaluacion_respuesta/b_estudios _complementarios/Resultados_Estudio_HSH_final125.pdf

Colombia, Ministerio de Salud y Protección Social \& Fondo de Población de las Naciones Unidas (2011b). Guía de Prevención VIH/Sida. Mujeres trans. Bogotá, Colombia: Autores. Extraído de http://unfpa.org.co/uploadUNFPA/file/PUB2011/VIH/TRANS.pdf

Colombia, Ministerio de Salud y Protección Social \& Fondo de Población de las Naciones Unidas (2012). Panorama del VIH/SIDA en Colombia 1983-2010: un análisis de situación. Bogotá, Colombia: Autores. Extraído de http://minsalud.gov.co/salud/Documents/observatorio_vih/documentos/monitoreo_evaluacion/1_vigilancia_salud_publica/b_estudio s_comportamiento/PANORAMAVIHCOL_WEB.pdf

Departamento de Salud y Servicios Humanos de EE. UU. (2016, Julio 8). El estigma prolonga la epidemia global del VIH entre los gais. InfoSIDA, Noticias del VIH/SIDA. Extraído de https://infosida.nih.gov/news/1686/el-estigma-prolonga-la-epidemia-global-del-vihentre-los-gais

Departamento de Salud y Servicios Humanos de EE. UU. (2019, Mayo 21). Profilaxis preexposición (PrEP). InfoSIDA, Prevención del VIH. Extraído de https://infosida.nih.gov/understanding-hiv-aids/fact-sheets/20/85/profilaxis-preexposicion--prep-

Díaz Murillo, M. P. (2002). El riesgo en salud: entre la visión del lego y el experto; una perspectiva sociocultural. Bogotá, Colombia: Universidad Nacional de Colombia, Facultad de Medicina.

Eribon, D. (2000). Identidades: reflexiones sobre la cuestión gay. Barcelona, España: Edicions Bellaterra.

Estrada-Montoya, J. H. (2014). Hombres que tienen sexo con hombres (HSH): reflexiones para la prevención y promoción de la salud. Revista Gerencia y Políticas de Salud, 13(26), 44-57. https://doi.org/10.11144/Javeriana.RGYPS13-26.htsh

Estrada, J. H. \& Vargas, L. (2011). Invisibles, excluidos y vulnerables: la infección VIH/SIDA entre hombres que tienen sexo con hombres (HSH). En M. Arrivillaga \& B. Useche (Dirs.), SIDA y sociedad: crítica y desafíos sociales frente a la epidemia (pp. 115-169). Bogotá, Colombia: Ediciones Aurora.

Fromm, E. (1955/1964). Psicoanálisis de la sociedad contemporánea: hacia una sociedad sana (6ª ed.; F. M. Torner, Trad.; Título original: The sane society). México DF, México: Fondo de Cultura Económica.

Gallego Villa, O. M. \& Barreiro de Motta, E. (2010). Análisis de los factores asociados a las relaciones de pareja homosexual (gays y lesbianas) en la ciudad de Bogotá. Suma Psicológica, 17, 69-81. Extraído de http://publicaciones.konradlorenz.edu.co/index.php/sumapsi/article/view/506/385

Garcia Abreu, A., Noguer, I. \& Cowgill, K. (2003/2004). El VIH/SIDA en países de América Latina: los retos futuros (Publicación Científica y Técnica $N^{\circ}$ 597; Título original: HIV/AIDS in Latin American Countries: The challenges ahead). Washington, DC: Organización Panamericana de la Salud/Banco Mundial.

García, F. E., García Escobar, C., Hein, H., Hernández, Á., Torres, P., Valdebenito, R. \& Vera, C. (2017). Relaciones de pareja homosexual y heterosexual: un estudio comparativo. Actualidades en Psicología, 31(122), 31-44. https://doi.org/10.15517/ap.v31i122.23346

García-Sánchez, I. (2004). Diferencias de género en el VIH/sida. Gaceta Sanitaria, 18(Supl 2), 47-54. https://doi.org/10.1157/13061994

Giménez, G. (1996). La identidad social o el retorno del sujeto en sociología. En L. I. Méndez y Mercado (Coord.), Identidad: análisis y teoría, simbolismo, sociedades complejas, nacionalismo y etnicidad: III Coloquio Paul Kirchhoff (pp. 11-24). México DF, México: Universidad Nacional Autónoma de México, Instituto de Investigaciones Antropológicas. Extraído de http://148.202.18.157/sitios/catedrasnacionales/material/2010a/cristina_palomar/1.pdf

Goetz, J. P. \& LeCompte M. D. (1984/1988). Etnografía y diseño cualitativo en investigación educativa (A. Ballesteros, Trad.; Título original: Ethnography and qualitative design in educational research). Madrid, España: Morata.

Gómez-Beneyto, M. (1978). La marginación del homosexual vista por un psicólogo-psiquiatra. En J. R. Enríquez (Comp.), El homosexual ante la sociedad enferma (pp. 39-46). Barcelona, España: Tusquets.

Granados-Cosme, J. A., Torres-Cruz, C. \& Delgado-Sánchez, G. (2009). La vivencia del rechazo en homosexuales universitarios de la Ciudad de México y situaciones de riesgo para VIH/SIDA. Salud Pública de México, 51, 482-488. https://doi.org/10.1590/S003636342009000600006

Guajardo, G. (2002). Contexto sociocultural del sexo entre varones. En C. F. Cáceres, M. Pecheny \& V. Terto Jr. (Eds.), SIDA y sexo entre hombres en América latina: vulnerabilidades, fortalezas, y propuestas para la acción - Perspectivas y reflexiones desde la salud pública, las ciencias sociales y el activismo (pp. 57-79). Lima, Perú: Universidad Peruana Cayetano Heredia/Programa Conjunto de las Naciones Unidas sobre el VIH/SIDA.

Juarez-Vílchez, J. P. \& Pozo, E. J. (2010). Percepciones sobre comportamientos sexuales de riesgo en personas que viven con VIH/SIDA y reciben tratamiento antirretroviral en Piura, Perú. Revista Peruana de Medicina Experimental y Salud Pública, 27, 31-37. https://doi.org/10.1590/S1726-46342010000100006

Lemaitre Ripoll, J. (2009). O amor em tempos de cólera: direitos LGTB na Colômbia [El amor en tiempos de cólera: derechos LGBT en Colombia]. Sur: Revista Internacional de Direitos Humanos, 6(11), 78-97. https://doi.org/10.1590/S1806-64452009000200005

Lluch Canut, M. T. (1999). Construcción de una escala para evaluar la salud mental positiva (Tesis de Doctorado, Universidad de Barcelona, España). Extraído de http://diposit.ub.edu/dspace/bitstream/2445/42359/1/E_TESIS.pdf

Lozano Santa, R. M. \& Talexio Paredes, R. E. (2011). Nivel de autoestima y uso de preservativo para prevenir el VIH/SIDA en adolescentes del AAHH El Porvenir Pampachica, Iquitos-2010. (Tesis de Licenciatura, Universidad Nacional de la Amazonía Peruana, Iquitos, Perú). Extraído de http://repositorio.unapiquitos.edu.pe/handle/UNAP/2250

Muñoz, C. B. (1996). Uruguay homosexual: culturas, minorías y discriminación desde una sociología de la homosexualidad. Montevideo, Uruguay: Trilce.

Organización Mundial de la Salud (2004). Informe sobre la salud en el mundo 2004 - cambiemos el rumbo de la historia. Genève, Suiza: Autor. Extraído de http://www.who.int/whr/2004/chapter1/es/index4.html 
Pisani, E., Brown, T., Saidel, T., Rehle, T. \& Caraël, M. (1998). Recopilación de datos necesarios sobre el comportamiento para los programas nacionales de VIH, SIDA e infecciones de transmisión sexual. Genève, Suiza: Programa Conjunto de las Naciones Unidas sobre el VIH/SIDA.

Posada, I. C. \& Gómez-Arias, R. D. (2007). Mercado y riesgo: escenarios de transmisión del VIH entre hombres que tienen sexo con otros hombres. Medellín, 1993-2006. Colombia Médica, 38, 222-236. https://doi.org/10.2510/colomb. med..v38i3.507

Programa Conjunto de las Naciones Unidas sobre el VIH/SIDA (1999). Infección por VIH-SIDA en Colombia: aspectos fundamentales, respuesta nacional y situación actual. Un balance histórico hacia el nuevo siglo. Bogotá, Colombia: Autor.

Salazar, X., Cáceres, C., Maiorana, A., Rosasco, A. M., Kegeles, S., Coates, T. \& NIMH Collaborative HIV/STI Prevention Trial Group (2006). Influencia del contexto sociocultural en la percepción del riesgo y la negociación de protección en hombres homosexuales pobres de la costa peruana. Cadernos de Saúde Pública, 22, 2097-2104. https://doi.org/10.1590/S0102-311X2006001000015

Seccional de Salud de Antioquia (1993). Significado del SIDA y la infección por VIH para grupos expuestos a un mayor riesgo de infección. Medellín, Colombia: Autor.

Strauss, A. \& Corbin, J. (1998/2002). Bases de la investigación cualitativa: técnicas y procedimientos para desarrollar la teoría fundamentada (E. Zimmerman, Trad.; Título original: Basics of qualitative research: Techniques and procedures for developing grounded theory). Medellín, Colombia: Editorial Universidad de Antioquia.

Stryker, S. (1987). The vitalization of symbolic interactionism. Social Psychology Quarterly, 50, 83-94. https://doi.org/10.2307/2786893

Ulin, P. R., Robinson, E. T. \& Tolley, E. E. (2005/2006). Investigación aplicada en salud pública: métodos cualitativos (Publicación Científica y Técnica $\mathrm{N}^{\circ} 614$; Título original: Qualitative methods in public health: A field guide for applied research). Washington, DC: Organización Panamericana de la Salud.

Fecha de recepción: Marzo de 2018.

Fecha de aceptación: Junio de 2019. 